\title{
Effect of Renal and Left Ventricular Function on Serial Pulmonary Arterial Pressure Changes after Device Closure of Atrial Septal Defect
}

\author{
Chul Shin $\left(\mathbb{D}\right.$, Young Won Yoon $\mathbb{D}^{\mathrm{D}}$, In-Soo Kim $\mathbb{D}$, Jong-Youn Kim $\mathbb{D}$, Pil-Ki Min $\mathbb{D}$, \\ Byoung Kwon Lee $\mathbb{D}$, Bum-Kee Hong $\mathbb{D}^{D}$, Se-Joong Rim $\mathbb{D}^{D}$, Hyuck Moon Kwon $\mathbb{D}$, \\ and Eui-Young Choi
}

Division of Cardiology, Heart Center, Gangnam Severance Hospital, Yonsei University College of Medicine, Seoul, Republic of Korea

Correspondence should be addressed to Eui-Young Choi; choi0928@yuhs.ac

Received 10 September 2020; Revised 27 December 2020; Accepted 30 December 2020; Published 18 January 2021

Academic Editor: Toshiko Nakai

Copyright $\odot 2021$ Chul Shin et al. This is an open access article distributed under the Creative Commons Attribution License, which permits unrestricted use, distribution, and reproduction in any medium, provided the original work is properly cited.

\begin{abstract}
Background. The age of candidates for device closure of atrial septal defect (ASD) has been increasing. Thus, concerns exist about dyspnea aggravation or atrial fibrillation development after device closure due to augmentation of left ventricular (LV) and left atrial (LA) preload. This study aimed to examine patterns and determinants of serial pulmonary arterial pressure and left ventricular filling pressure changes after device closure of ASD. Methods. Among the 86 consecutive patients who underwent percutaneous device closure of ASD, those with end-stage renal disease or those without pre- or postprocedural Doppler data were excluded. The clinical, transesophageal, and transthoracic echocardiographic findings of 78 patients were collected at baseline, one-day postprocedure, and one-year follow-up. Results. The mean age of study patients was $49.8 \pm 15.0$ years, and the average maximal defect diameter and device size were $20.2 \pm 6.0 \mathrm{~mm}$ and $23.8 \pm 6.4 \mathrm{~mm}$. Four patients (5.6\%) underwent new-onset atrial fibrillation, and five patients (6.4\%) took diuretics within one-year after closure. Some patients $(n=21 ; 27 \%)$ exhibited paradoxically increased tricuspid regurgitant velocity (TRV) one-day postprocedure; they also were older with lower e', glomerular filtration rate, and LV ejection fraction and a higher LA volume index. However, even in these patients, TRV deceased below baseline levels one-year later. Both E/e' and LA volume index significantly increased immediately after device closure, but all decreased one-year later. Larger defect size and higher TRV were significantly correlated with immediate E/e' elevation. Conclusion. In older, renal, diastolic, and systolic dysfunctional patients with larger LA and scheduled for larger device implantation, peri-interventional preload reduction therapy would be beneficial.
\end{abstract}

\section{Introduction}

The age of candidates for device closure of atrial septal defect (ASD) has been increasing. Especially in older patients, concerns exist about dyspnea aggravation [1] or atrial fibrillation development after device closure due to the augmentation of left ventricular (LV) and left atrial (LA) preload $[2,3]$. Although recent studies showed that the long-term outcome of device closure is quite good even in older patients, vulnerable patients must be identified to prepare them for periprocedural preload reduction management. Thus, in this study, we sought to examine serial hemodynamic changes, including pulmonary arterial systolic pressure, LV filling pressure, stroke volume, and LA volume, after device closure of ASD. In addition, factors contributing to adverse hemodynamic changes from immediately after the procedure to one-year follow-up were determined.

\section{Materials and Methods}

2.1. Study Population and Clinical Follow-Up. The database of a single center of patients who underwent percutaneous device closure of ASD was analyzed. Patients underwent transthoracic echocardiography (TTE) and transesophageal echocardiography (TEE) before the device 
closure procedure. Among the 86 consecutive patients who underwent percutaneous device closure of ASD, patients with end-stage renal disease or patients without pre- or postprocedural Doppler data were excluded. In total, the clinical, transesophageal, and transthoracic echocardiographic findings of 78 patients were collected at baseline, one day (immediately) after the procedure, and one-year follow-up (Figure 1). All patients underwent blood chemistry and cell blood count analysis. Estimated glomerular filtration rate (eGFR) was calculated using the Chronic Kidney Disease Epidemiology Collaboration creatinine equation [4]. Clinical characteristics, medical history, laboratory findings, medications, and echocardiographic data were collected and used for analysis. The study protocol was approved by the institutional review board of Gangnam Severance hospital (3-2020-0026), and the need for written informed consent was waived due to the study's retrospective design.

\subsection{Echocardiography at Baseline and after Device Closure.} Each patient underwent a complete standard TTE. TTE findings were collected at baseline, one-day (immediately) postclosure, and one-year follow-up. LV dimensions and septal and posterior wall thickness were measured at enddiastole and end-systole in the two-dimensional (2D) parasternal long- or short-axis views. LV ejection fraction was calculated using the modified Quinones' method [5]. LV mass was measured by Devereux's methods as recommended by the American Society of Echocardiography [5]. LA volume was measured using the prolate ellipsoidal method at the point of LV end-systole at maximum LA size. From the apical window, a $1 \mathrm{~mm}$ pulsed Doppler sample volume was placed at the mitral valve tip, and mitral flow velocities from 5-10 cardiac cycles were recorded. Peak early (E) and late (A) mitral inflow velocities were also measured. Mitral annular velocity was measured by tissue Doppler imaging using the pulsed-wave Doppler mode. The filter was set to exclude high-frequency signals, and the Nyquist limit was adjusted to a range of $15-20 \mathrm{~cm} / \mathrm{s}$. Gain and sample volume were minimized to allow for a clear tissue signal with minimal background noise. Systolic (S') and early (e') and late diastolic velocities of the mitral annulus were measured from the apical four-chamber view with a sample volume $(2-5 \mathrm{~mm})$ placed at the septal corner of the mitral annulus. Peak velocity of tricuspid regurgitation was measured. Pulmonary arterial systolic pressure (PASP) was calculated as follows: $4 \times$ tricuspid regurgitant velocity $(\mathrm{TRV})^{2}+$ right atrial pressure, where right atrial pressure was estimated according to inferior vena cava diameter and its respiratory variations [6]. E/e' divided by stroke volume was defined as LV end-diastolic elastance index [7]. During TEE, multiplane $2 \mathrm{D}$ and zoomed $3 \mathrm{D}$ images were acquired, and both long- and short-axis diameters of the ASD were measured. Area was calculated as long-axis diameter multiplied by short-axis diameter and 3.14. In multiple defects, the sum of all diameters or areas was used. The margins to the aorta, posterior wall, superior vena cava, and inferior vena cava rims were also evaluated.
2.3. Device Implantation and Periprocedural Imaging. Transcatheter ASD closure was performed, as described previously [8], using various types of septal occluders (Amplatzer/Cocoon/Figullar Flex II/Gore-Helix/Occlutech). Before the procedure, the pulmonary-to-systemic blood flow ratio and pulmonary artery pressure were evaluated using cardiac catheterization. During the procedure, 3D-TEE or intracardiac echocardiography (ICE) were performed to guide accurate device implantation and ensure successful device closure. After the procedure, all patients received $100 \mathrm{mg}$ /day aspirin for at least 6 months and $75 \mathrm{mg}$ clopidogrel for 3 months. Other medications, such as diuretics and antihypertensive medications, were continued.

2.4. Statistical Analysis. Clinical characteristics and echocardiographic parameters are presented as the means \pm standard deviation for continuous variables and the numbers (percentage) for categorical variables. Correlation analysis was performed between continuous variables using the Pearson correlation coefficient. Comparisons of clinical and echocardiographic findings between two groups were performed using the independent $t$-test. Nonparametric comparisons of two groups were done by Mann-Whitney $U$ test. Serial changes in echocardiographic parameters were assessed using repeated ANOVA or the paired $t$-test. Variables with $P$ values less than 0.05 in univariate analysis were included in the multivariable linear or logistic regression analysis. All the analyses were performed using SPSS (version 25.0, IBM, USA), and $P$ values less than 0.05 were considered significant.

\section{Results}

3.1. Baseline Characteristics, Echocardiographic Parameters, and Hemodynamic Findings. The mean age of enrolled patients was $49.8 \pm 15.0$ (range, 16-77) years, and $51(65 \%)$ were female. The average body mass index was $22.5 \pm 3.0 \mathrm{~kg} /$ $\mathrm{m}^{2}$, and the average eGFR was $99.4 \pm 20.0 \mathrm{~mL} / \mathrm{min}$. Mean LV ejection fraction and LV mass index were $66.1 \pm 6.4 \%$ and $63.3 \pm 16.3 \mathrm{~g} / \mathrm{m}^{2}$, respectively. Among them, 4 patients had more than one defects. Measured average defect maximal diameter was $20.2 \pm 6.0 \mathrm{~mm}$, and defect area was $2.57 \pm 1.52 \mathrm{~cm}^{2}$ on preprocedural TEE. The calculated Qp/Qs ratio during right side catheterization before device implantation was $2.52 \pm 0.85$. Sixty-four patients underwent TEE, and 14 patients underwent ICE for periprocedural guidance. The average size of the septal occluder was $23.8 \pm 6.4 \mathrm{~mm}$. Baseline characteristics are described in Table 1.

3.2. Clinical Problems after Device Closure. Six patients had history of persistent atrial fibrillation or documented paroxysmal atrial fibrillation. Among the rest $(n=72)$, four patients $(4 / 72,5.6 \%)$ underwent newly developed or detected atrial fibrillation within one year after device closure. Although it is not statistically significant due to small number of the patients, the patients with new-onset atrial fibrillation after closure had tendency of older age, 


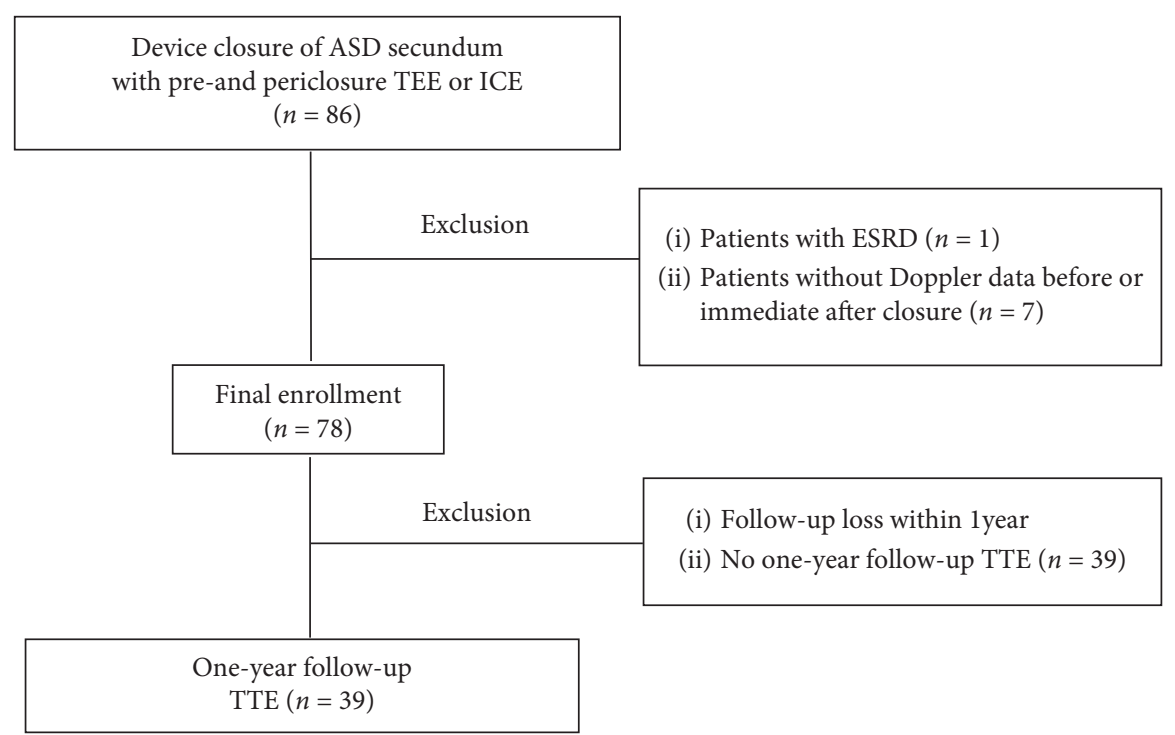

FIGURE 1: Schematic diagram of study patient selection process. ASD, atrial septal defect; ESRD, end-stage renal disease; TTE, transthoracic echocardiography; TEE, transesophageal echocardiography; ICE, intracardiac echocardiography.

TABle 1: Baseline clinical characteristics and echocardiographic measurements in patients with ASD.

\begin{tabular}{|c|c|}
\hline Variable $(n=78)$ & Parameter \\
\hline Age, years & $49.8 \pm 15.0$ \\
\hline Female, $n(\%)$ & $51(65)$ \\
\hline Body mass index, $\mathrm{kg} / \mathrm{m}^{2}$ & $22.4 \pm 3.0$ \\
\hline Systolic blood pressure, $\mathrm{mmHg}$ & $116.5 \pm 15.7$ \\
\hline Blood urea nitrogen, $\mathrm{mg} / \mathrm{dL}$ & $15.1 \pm 4.4$ \\
\hline Creatinine, $\mathrm{mg} / \mathrm{dL}$ & $0.75 \pm 0.16$ \\
\hline $\mathrm{eGFR}, \mathrm{mL} / \mathrm{min} / 1.73 \mathrm{~m}^{2}$ & $99.8 \pm 18.8$ \\
\hline Atrial fibrillation, $n(\%)$ & $6(8)$ \\
\hline $\mathrm{LV}$ mass index, $\mathrm{g} / \mathrm{m}^{2}$ & $63.3 \pm 16.3$ \\
\hline LA volume index, $\mathrm{mL} / \mathrm{m}^{2}$ & $30.5 \pm 12.5$ \\
\hline LV ejection fraction, $\%$ & $66.1 \pm 6.4$ \\
\hline Stroke volume, $\mathrm{mL}$ & $52.1 \pm 11.2$ \\
\hline $\mathrm{TRV}, \mathrm{m} / \mathrm{sec}$ & $2.73 \pm 0.48$ \\
\hline PASP, mmHg & $37.0 \pm 12.3$ \\
\hline PVR, Wood unit & $1.47 \pm 0.31$ \\
\hline $\mathrm{e}^{\prime}, \mathrm{cm} / \mathrm{s}$ & $9.3 \pm 3.2$ \\
\hline $\mathrm{E} / \mathrm{e}^{\prime}$ & $9.0 \pm 3.5$ \\
\hline More than single defects, $n(\%)$ & $4(5)$ \\
\hline ASD maximal defect diameter, $\mathrm{mm}$ & $20.2 \pm 6.0$ \\
\hline ASD defect area, $\mathrm{cm}^{2}$ & $2.57 \pm 1.52$ \\
\hline ASD device diameter, $\mathrm{mm}$ & $23.8 \pm 6.4$ \\
\hline Qp/Qs ratio & $2.52 \pm 0.85$ \\
\hline Procedure guidance by TEE/ICE & $64 / 14$ \\
\hline Amplatzer, Abbott/Cocoon, Vascular Innovations/Figulla Flex II, Occlutech/Helex, Gore & $41 / 33 / 3 / 1$ \\
\hline
\end{tabular}

lower eGFR, e', and LV ejection fraction, and larger defect size, LA volume index, and E/e. We could not find a case with development of significant pulmonary edema; however, five patients underwent taking diuretics after device closure. They had higher prevalence of atrial fibrillation, E/e', and lower eGFR, tendency of older age, lower eGFR, e', and higher LA volume index and E/e' without significance (Table 2).
3.3. Serial Changes in LV Filling Pressure, LA Volume, and Stroke Volume. At baseline, E/e' and LA volume index were $9.0 \pm 3.5$ and $30.5 \pm 12.5 \mathrm{~mL} / \mathrm{m}^{2}$, respectively. One day after the procedure, these values significantly increased to $11.4 \pm 4.0$ and $32.5 \pm 12.1 \mathrm{~mL} / \mathrm{m}^{2}$, respectively, due to increased LV filling from occlusion of a left-to-right shunt. LV stroke volume increased from $52.1 \pm 11.2 \mathrm{~mL}$ to $61.5 \pm 19.4 \mathrm{~mL}$ the day after the procedure. At one-year 
TABLE 2: Comparisons between groups according to new-onset atrial fibrillation after device closure and diuretics use.

\begin{tabular}{|c|c|c|c|c|c|c|}
\hline Variable & $\begin{array}{l}\text { New-onset AF after closure } \\
\qquad(n=4)\end{array}$ & $\begin{array}{l}{ }^{*} \text { No AF } \\
(n=68)\end{array}$ & $\begin{array}{c}{ }^{\dagger} P \\
\text { value }\end{array}$ & $\begin{array}{l}\text { Diuretics use } \\
\quad(n=5)\end{array}$ & $\begin{array}{l}\text { No diuretics use } \\
(n=73)\end{array}$ & $\begin{array}{c}{ }^{\dagger} P \\
\text { value }\end{array}$ \\
\hline Age, years & $60.0 \pm 10.5$ & $47.9 \pm 14.9$ & 0.113 & $58.8 \pm 13.7$ & $49.2 \pm 15.0$ & 0.124 \\
\hline Female, $n(\%)$ & $2(50)$ & $45(66)$ & 0.509 & $3(60)$ & $48(66)$ & 0.794 \\
\hline Past history of AF, $n(\%)$ & & & & $4(80)$ & $2(3)$ & $<0.001$ \\
\hline $\mathrm{eGFR}, \mathrm{mL} / \mathrm{min} / 1.73 \mathrm{~m}^{2}$ & $90.0 \pm 10.6$ & $102.8 \pm 17.4$ & 0.085 & $80.8 \pm 14.1$ & $101.2 \pm 18.4$ & 0.018 \\
\hline $\begin{array}{l}\text { Maximal defect diameter, } \\
\mathrm{mm}\end{array}$ & $23.0 \pm 5.9$ & $20.4 \pm 6.0$ & 0.308 & $20.0 \pm 4.7$ & $20.2 \pm 6.2$ & 0.886 \\
\hline Defect area, $\mathrm{mm}^{2}$ & $298.1 \pm 110.8$ & $262.1 \pm 155.8$ & 0.433 & $264.9 \pm 151.4$ & $256.5 \pm 153.5$ & 0.975 \\
\hline Device diameter, $\mathrm{mm}$ & $26.0 \pm 3.7$ & $24.0 \pm 6.5$ & 0.491 & $22.8 \pm 4.6$ & $23.9 \pm 6.5$ & 0.779 \\
\hline LV mass index, $\mathrm{g} / \mathrm{m}^{2}$ & $65.9 \pm 17.5$ & $62.8 \pm 16.5$ & 0.525 & $55.6 \pm 14.4$ & $63.9 \pm 16.4$ & 0.295 \\
\hline LA volume index, $\mathrm{mL} / \mathrm{m}^{2}$ & $35.8 \pm 6.5$ & $28.1 \pm 9.3$ & 0.065 & $39.4 \pm 20.4$ & $29.9 \pm 11.7$ & 0.205 \\
\hline LV ejection fraction, $\%$ & $62.3 \pm 4.3$ & $66.3 \pm 6.6$ & 0.198 & $65.4 \pm 5.3$ & $66.2 \pm 6.5$ & 0.772 \\
\hline Stroke volume, $\mathrm{mL}$ & $51.6 \pm 9.4$ & $53.1 \pm 10.7$ & 0.832 & $40.7 \pm 14.9$ & $53.0 \pm 10.5$ & 0.038 \\
\hline $\mathrm{TRV}, \mathrm{m} / \mathrm{sec}$ & $2.63 \pm 0.36$ & $2.72 \pm 0.49$ & 0.765 & $2.89 \pm 0.56$ & $2.72 \pm 0.48$ & 0.004 \\
\hline PASP, $\mathrm{mmHg}$ & $36.8 \pm 4.8$ & $36.3 \pm 12.6$ & 0.549 & $45.5 \pm 12.7$ & $36.4 \pm 12.2$ & 0.306 \\
\hline PVR, Wood unit & $1.24 \pm 0.43$ & $1.45 \pm 0.27$ & 0.336 & $1.91 \pm 0.41$ & $1.44 \pm 0.28$ & 0.088 \\
\hline $\mathrm{e}^{\prime}, \mathrm{cm} / \mathrm{s}$ & $8.8 \pm 3.5$ & $9.3 \pm 3.3$ & 0.787 & $8.9 \pm 1.7$ & $9.3 \pm 3.3$ & 0.974 \\
\hline $\mathrm{E} / \mathrm{e}^{\prime}$ & $10.7 \pm 6.9$ & $8.5 \pm 3.0$ & 0.681 & $11.5 \pm 3.1$ & $8.8 \pm 3.4$ & 0.038 \\
\hline
\end{tabular}

AF, atrial fibrillation; see abbreviations in Table $1 .{ }^{*}$ Patients with pre-existing AF were excluded; ${ }^{\dagger}$ Mann-Whitney test for nonparametric comparisons.

follow-up, E/e' and LA volume index decreased by $9.8 \pm 3.0$ and $27.8 \pm 8.6 \mathrm{~mL} / \mathrm{m}^{2}$, which was accompanied by a further increase in LV stroke volume (Figure 2). Immediate E/e' changes after device closure $\left(\Delta \mathrm{E} / \mathrm{e}^{\prime}\right.$-immediate) were significantly correlated with immediate LA volume index change $(r=0.424 ; P<0.001)$. ASD area, maximal defect diameter, and implanted device diameter were significantly correlated with $\Delta \mathrm{E} / \mathrm{e}^{\prime}$-immediate but not with age. Higher TRV and lower baseline E/e' were correlated with $\Delta \mathrm{E} / \mathrm{e}^{\prime}$ immediate (Table 3). In multivariate analysis, TRV and baseline $\mathrm{E} / \mathrm{e}^{\prime}$ were related to $\Delta \mathrm{E} / \mathrm{e}^{\prime}$-immediate.

3.4. Serial Changes in Pulmonary Arterial Pressure after Device Closure. Of the 78 patients enrolled in the study, in two patients, pre- or post-TRV were not measurable and 39 underwent TTE at one-year follow-up. The average PASP decreased approximately $-6.15 \pm 11.6 \mathrm{mmHg}$ immediately after closure, but some patients $(n=21 ; 28 \%)$ paradoxically exhibited increase in PASP. Patients with immediately increased PASP were older, with lower eGFR, LV ejection fraction, and e' and higher LA volume index (Table 4). In multivariate analysis for increased PASP-immediate, LV ejection fraction was significantly related to PASP increase immediately after closure. When serially followed, these patients also showed serially decreased PASP one-year later below the baseline level, similar to patients with initially decreased PASP (Figure 3). In patients with immediately increased PASP, accompanying increase in LA size was significantly blunted compared to decreased PASP group (Figure 4).

\section{Discussion}

According to our study results, we found some patients underwent new-onset atrial fibrillation and took diuretics within one-year after device closure. In addition, some patients who underwent device closure of ASD exhibited immediate PASP elevation even after transpulmonary arterial flow was reduced by blocking left-to-right shunt flow. This finding may be from immediately elevated pulmonary capillary wedge pressure due to increased LV filling flow to the noncompliant left ventricle or volume overload to the noncompliant left atrium (perhaps from not only the muscular part but also the device-covered noncompliant part) [9]. This speculation was supported by our findings that impaired LV relaxation (as represented by lower e') and systolic function, and higher LA volume index was found in the initial increased PASP group. This finding was more predominant in older patients with lower eGFR, suggesting that these patients need peri-interventional preload reducing medication, especially those with impaired diastolic function and larger LA size. When elevated LV filling pressure measured by E/e' was seen after device closure, larger defect size was more related to LV filling pressure elevation. Thus, especially in patients scheduled to close a larger defect, physicians should be cautious to avoid postinterventional pulmonary edema or exertional dyspnea aggravation. When considering the poor correlation between delta PASP elevation and delta E/e' elevation after device closure, increased PASP would be contributed by not only elevated LV filling pressure [10] but also LA noncompliance, which is also contributed by the device itself in the LA septum. An immediate increase in E/e' was significantly correlated with increase in LA volume index. However, at one-year followup, decreased LV filling pressure was accompanied by decreased LA volume index. When examining serial changes at one-year follow-up, we found that all patients showed decreased PASP below the baseline level regardless of the immediate PASP response, even in the paradoxically increased PASP group. Thus, despite the concern of device closure in older patients with renal dysfunction, these findings support that long-term hemodynamic response 


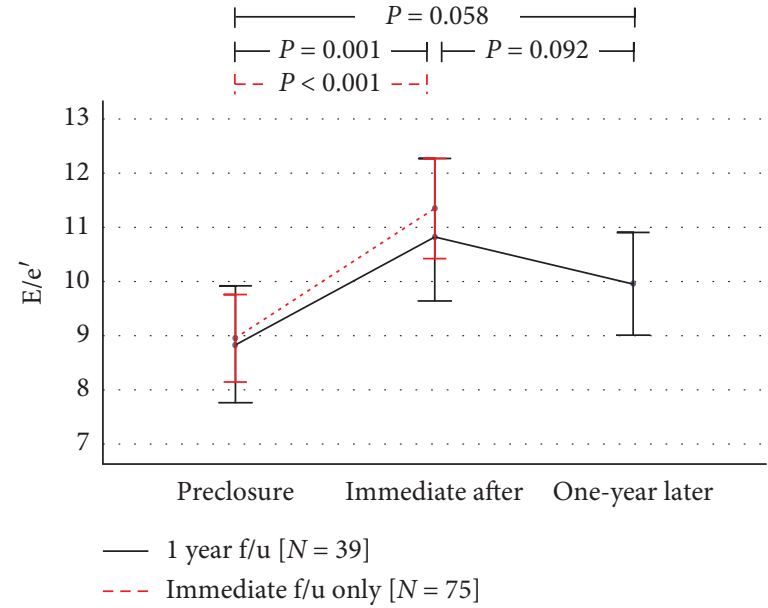

(a)

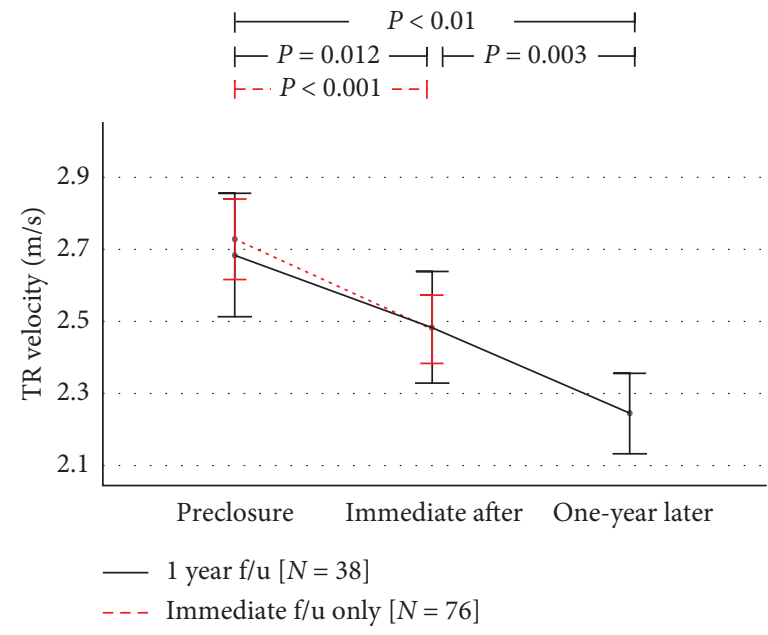

(c)

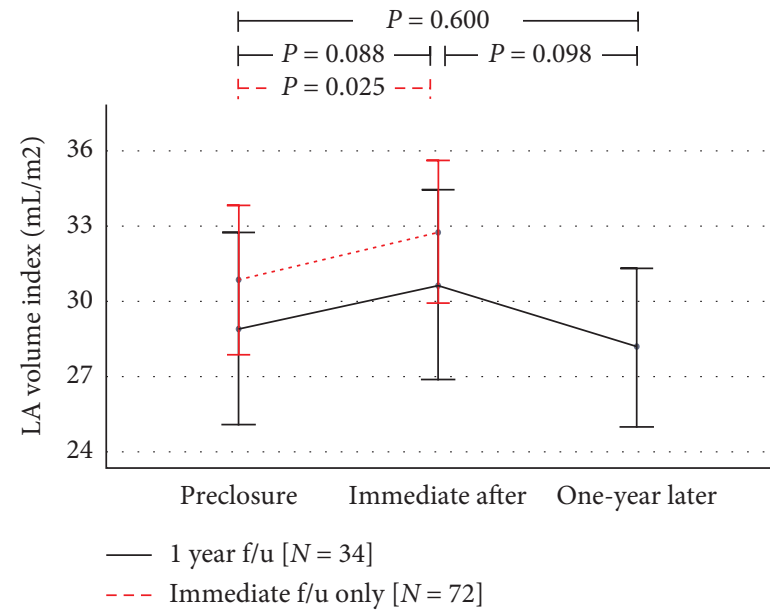

(b)

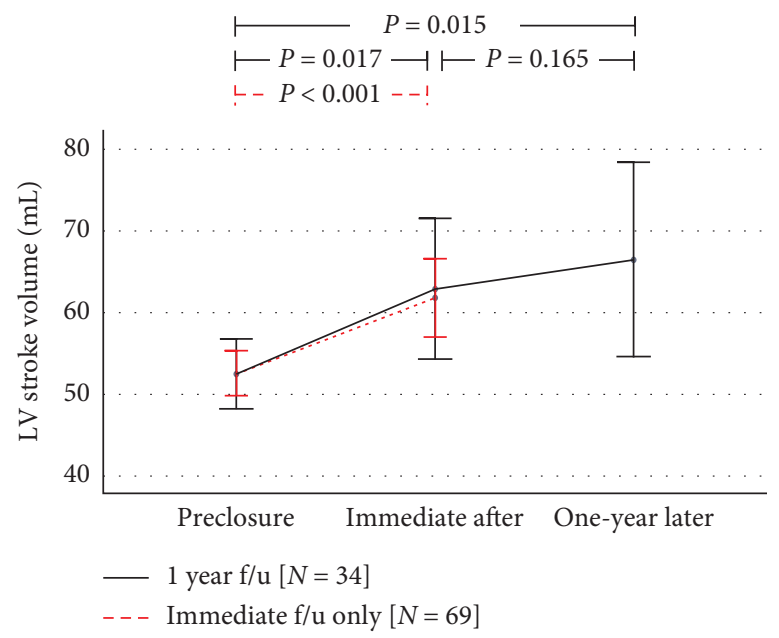

(d)

Figure 2: Serial changes in E/e' (a), left atrial (LA) volume index (b), tricuspid regurgitant (TR) velocity (c), and left ventricular (LV) stroke volume (d) from preclosure to one-year follow-up $(n=39)$. Blue dotted line represents comparisons between preclosure state and immediately after (one-day after closure) follow-up only $(n=78)$. Bars represent $95 \%$ confidence intervals.

TABLE 3: Correlations of immediate E/e' changes after device closure of an ASD.

\begin{tabular}{|c|c|c|}
\hline Variable & Pearson's correlation coefficient ( $r$ value) & $P$ value \\
\hline Age, years & 0.039 & 0.742 \\
\hline Male & -0.118 & 0.331 \\
\hline Body mass index, $\mathrm{m}^{2}$ & -0.168 & 0.123 \\
\hline Systolic blood pressure, $\mathrm{mmHg}$ & -0.042 & 0.738 \\
\hline Blood urea nitrogen, mg/dL & 0.112 & 0.338 \\
\hline Creatinine, $\mathrm{mg} / \mathrm{dL}$ & 0.183 & 0.115 \\
\hline $\mathrm{eGFR}, \mathrm{mL} / \mathrm{min} / 1.73 \mathrm{~m}^{2}$ & -0.110 & 0.349 \\
\hline ASD maximal defect diameter, $\mathrm{mm}$ & 0.248 & 0.032 \\
\hline ASD defect area, $\mathrm{cm}^{2}$ & 0.309 & 0.008 \\
\hline ASD device diameter, $\mathrm{mm}$ & 0.248 & 0.033 \\
\hline Qp/Qs ratio & 0.217 & 0.103 \\
\hline $\mathrm{LV}$ mass index, $\mathrm{g} / \mathrm{m}^{2}$ & 0.080 & 0.519 \\
\hline LA volume index, $\mathrm{mL} / \mathrm{m}^{2}$ & -0.078 & 0.509 \\
\hline LV ejection fraction, $\%$ & 0.191 & 0.103 \\
\hline Stroke volume, $\mathrm{mL}$ & -0.137 & 0.251 \\
\hline $\mathrm{TRV}, \mathrm{m} / \mathrm{sec}$ & 0.332 & 0.004 \\
\hline Preclosure E/e' & -0.359 & 0.002 \\
\hline Preclosure Ed & -0.227 & 0.055 \\
\hline$\Delta \mathrm{LA}$ volume index-immediate & 0.424 & $<0.001$ \\
\hline Immediate LV Ed & 0.480 & $<0.001$ \\
\hline
\end{tabular}

ASD, atrial septal defect; e', early diastolic septal mitral annular velocity; Ed, end-diastolic elastance; E/e', ratio of early mitral inflow velocity to e'; eGFR, estimated glomerular filtration rate; LA, left atrial; LV, left ventricular; TRV, tricuspid regurgitant velocity. 
TABle 4: Comparison between patients with increased PASP and decreased PASP immediately after device closure of ASDs*.

\begin{tabular}{|c|c|c|c|}
\hline Variable & $\begin{array}{l}\text { PASP decrease } \\
\quad(n=55)\end{array}$ & $\begin{array}{l}\text { PASP increase } \\
\quad(n=21)\end{array}$ & $P$ value \\
\hline Age, years & $47.7 \pm 14.4$ & $56.4 \pm 15.2$ & 0.022 \\
\hline Female, $n(\%)$ & $36(66)$ & $14(67)$ & 0.921 \\
\hline Body surface area, $\mathrm{m}^{2}$ & $1.62 \pm 0.14$ & $1.57 \pm 0.18$ & 0.288 \\
\hline Body mass index, $\mathrm{kg} / \mathrm{m}^{2}$ & $22.3 \pm 2.9$ & $22.3 \pm 3.1$ & 0.986 \\
\hline Systolic blood pressure, $\mathrm{mmHg}$ & $116.7 \pm 13.7$ & $115.1 \pm 19.7$ & 0.027 \\
\hline Atrial fibrillation, $n(\%)$ & $4(7)$ & $2(10)$ & 0.745 \\
\hline Blood urea nitrogen, $\mathrm{mg} / \mathrm{dL}$ & $14.4 \pm 3.7$ & $16.7 \pm 5.8$ & 0.046 \\
\hline Creatinine, $\mathrm{mg} / \mathrm{dL}$ & $0.74 \pm 0.15$ & $0.80 \pm 0.20$ & 0.168 \\
\hline $\mathrm{eGFR}, \mathrm{mL} / \mathrm{min} / 1.73 \mathrm{~m}^{2}$ & $102.6 \pm 17.4$ & $91.3 \pm 20.3$ & 0.018 \\
\hline Maximal defect diameter, $\mathrm{mm}$ & $20.1 \pm 6.2$ & $21.1 \pm 5.7$ & 0.522 \\
\hline Defect area, $\mathrm{mm}^{2}$ & $253.0 \pm 144.1$ & $263.5 \pm 156.4$ & 0.794 \\
\hline Device diameter, $\mathrm{mm}$ & $23.6 \pm 6.5$ & $25.0 \pm 5.9$ & 0.411 \\
\hline Qp/Qs ratio & $2.46 \pm 0.82$ & $2.79 \pm 0.95$ & 0.214 \\
\hline $\mathrm{LV}$ mass index, $\mathrm{g} / \mathrm{m}^{2}$ & $62.6 \pm 14.1$ & $64.4 \pm 21.3$ & 0.673 \\
\hline LA volume index, $\mathrm{mL} / \mathrm{m}^{2}$ & $29.0 \pm 12.5$ & $35.4 \pm 11.7$ & 0.046 \\
\hline LV ejection fraction, $\%$ & $67.4 \pm 5.9$ & $63.4 \pm 7.0$ & 0.015 \\
\hline Stroke volume, $\mathrm{mL}$ & $52.4 \pm 11.2$ & $51.9 \pm 11.7$ & 0.869 \\
\hline $\mathrm{TRV}, \mathrm{m} / \mathrm{sec}$ & $2.83 \pm 0.46$ & $2.48 \pm 0.45$ & 0.004 \\
\hline PASP, $\mathrm{mmHg}$ & $39.2 \pm 12.7$ & $31.6 \pm 9.6$ & 0.016 \\
\hline PVR, Wood unit & $1.49 \pm 0.27$ & $1.42 \pm 0.42$ & 0.425 \\
\hline $\mathrm{e}^{\prime}, \mathrm{cm} / \mathrm{s}$ & $9.8 \pm 3.0$ & $7.7 \pm 3.4$ & 0.013 \\
\hline $\mathrm{E} / \mathrm{e}^{\prime}$ & $8.65 \pm 3.29$ & $10.10 \pm 3.94$ & 0.120 \\
\hline $\begin{array}{l}\text { Amplatzer, Abbott/Cocoon, Vascular Innovations/Figulla Flex II, Occlutech/ } \\
\text { Helex, Gore }\end{array}$ & $29 / 23 / 3 / 0$ & $10 / 10 / 0 / 1$ & 0.266 \\
\hline
\end{tabular}

${ }^{*}$ In two patients, pre- or post-TRV were not measurable. ASD, atrial septal defect; e', early diastolic septal mitral annular velocity; E/e', ratio of early mitral inflow velocity to e'; eGFR, estimated glomerular filtration rate; LA, left atrial; LV, left ventricular; PASP, pulmonary arterial systolic pressure; PVR, pulmonary vascular resistance; TRV, tricuspid regurgitant velocity.

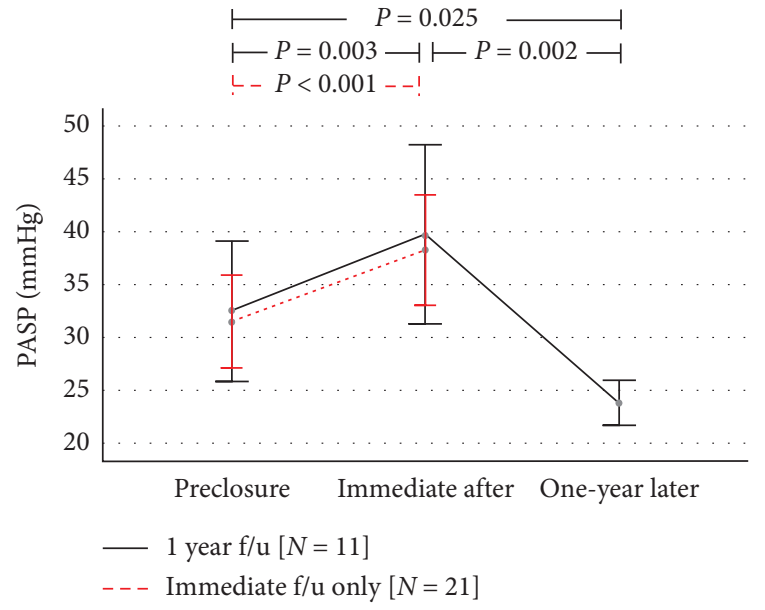

(a)

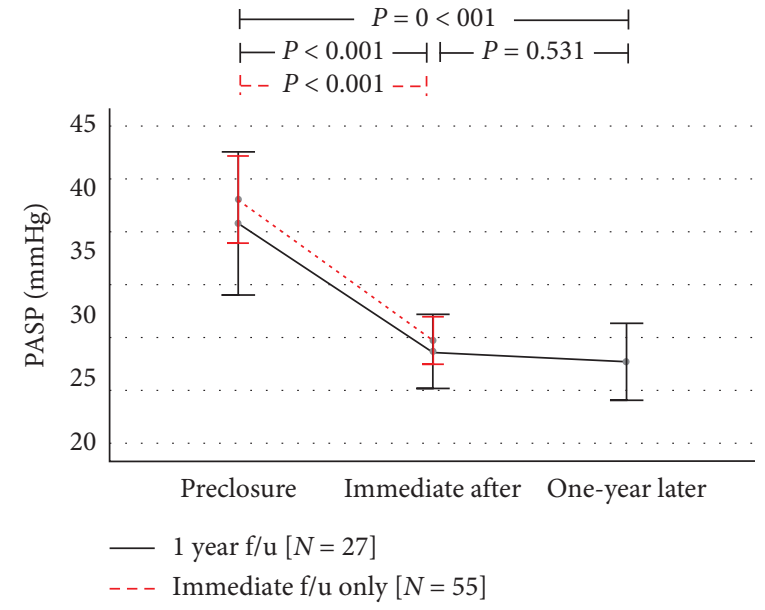

(b)

FIGURE 3: Serial changes in pulmonary arterial systolic pressure (PASP) from preclosure stage to one-year follow-up by immediate PASP response group: (a) immediately increased PASP group; (b) immediately decreased PASP group. Blue dotted line represents comparisons between preclosure state and immediately after (one-day after closure) follow-up only. Bars represent $95 \%$ confidence intervals.

would be favorable in older patients. Therefore, with optimal preload reduction and volume control during and immediately after the procedure, device closure of ASD can be recommended even in vulnerable patients [11].

This study has some limitations. Although we found a significant proportion of patients underwent paradoxically increase in PASP, especially in older patients, with renal dysfunction, impaired LV relaxation, and larger LA, the contribution of the device on the LA side could not be fully determined. We could only assume that, with larger LA and marginal compliance, adding a prosthesis may worsen LA compliance. Although we could not find any difference in device type, future studies are warranted to determine how to reduce worsening LA compliance when selecting device size, device type, and deployment method. 


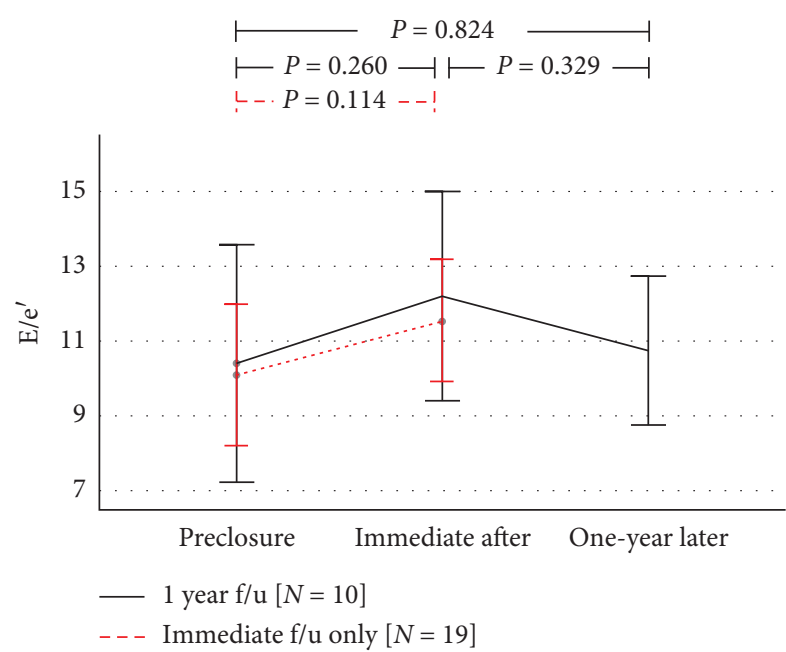

(a)

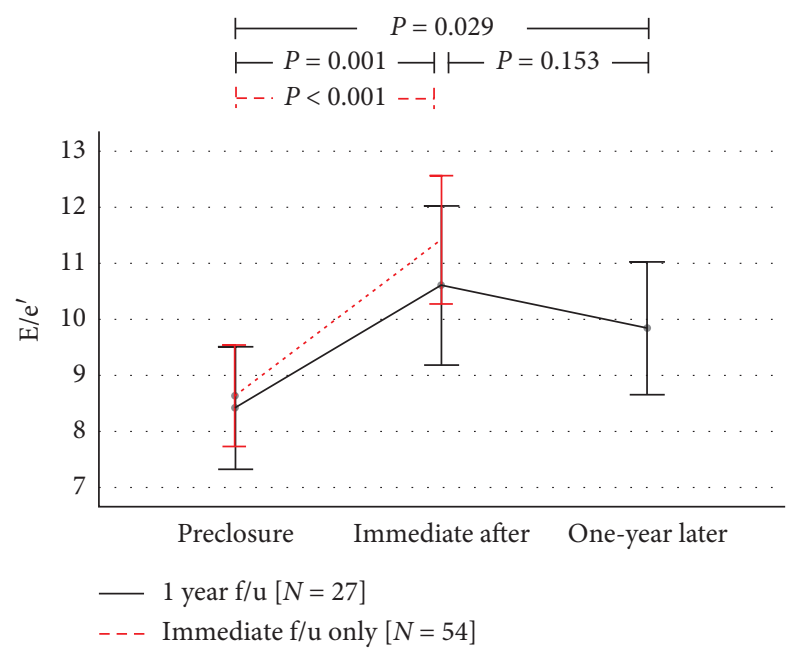

(c)

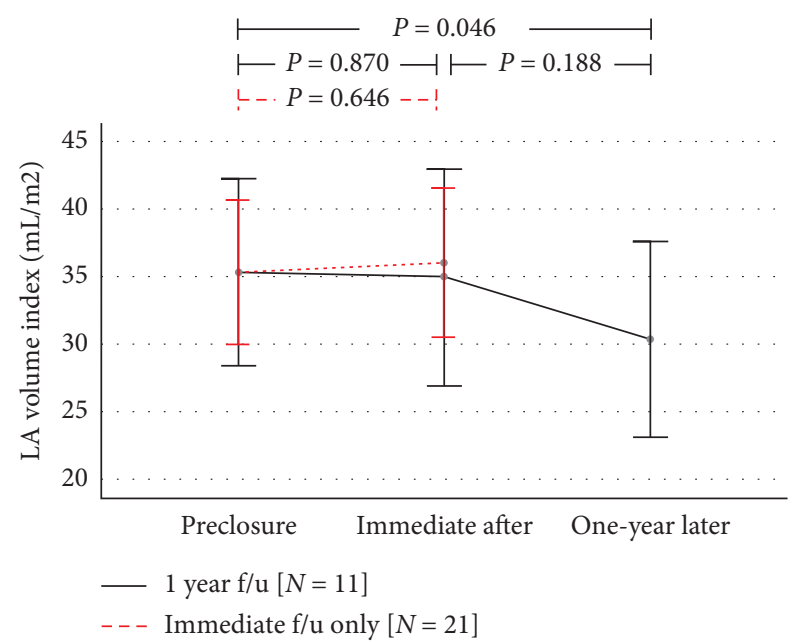

(b)

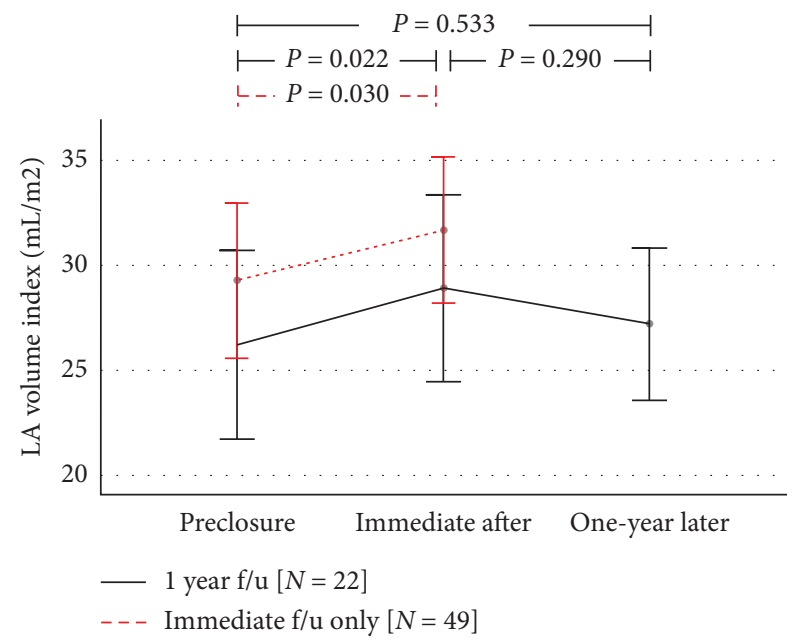

(d)

FIgURE 4: Serial changes in E/e', left atrial (LA) volume index, tricuspid regurgitant (TR) velocity, and left ventricular (LV) stroke volume from preclosure stage to one-year follow-up in $(\mathrm{a}, \mathrm{b})$ immediately increased PASP group and $(\mathrm{c}, \mathrm{d})$ immediately decreased PASP group. Blue dotted line represents comparisons between preclosure state and immediately after (one-day after closure) follow-up only. Bars represent $95 \%$ confidence intervals.

\section{Conclusions}

In older patients with impaired LV relaxation, systolic dysfunction, renal dysfunction, or larger LA size, PASP could paradoxically increase after device closure of ASD due to the immediate volume overload to a noncompliant left ventricle and left atrium. Therefore, periprocedural preload manipulation, such as diuretics, is recommended in patients with these risk factors who are scheduled to close a larger defect. However, after the immediate periprocedural period, TRV, LA volume index, and E/e' continuously decrease, suggesting the favorable effects of ASD closure even in these high-risk patients. The risk for significant symptoms of heart failure is small with due precautions as none of the patients in this cohort experienced any.

\section{Abbreviations}

ASD: Atrial septal defect

E: $\quad$ Peak early diastolic mitral inflow velocity

e': $\quad$ Peak early diastolic mitral annular velocity

eGFR: Estimated glomerular filtration rate

ICE: Intracardiac echocardiography

LA: Left atrial

LV: Left ventricular

LVOT: LV outflow tract

PASP: Pulmonary arterial systolic pressure

TEE: Transesophageal echocardiography

TTE: Transthoracic echocardiography

TRV: Tricuspid regurgitant velocity

2D: $\quad$ Two dimensional

3D: Three dimensional. 


\section{Data Availability}

The datasets used and/or analyzed during the current study are available from the corresponding author on reasonable request.

\section{Ethical Approval}

All procedures performed in studies involving human participants were in accordance with the ethical standards of the institutional research committee and with the 1964 Helsinki declaration and its later amendments or comparable ethical standards. The study protocol was approved by the Institutional Review Board of Gangnam Severance Hospital (3-2020-0026).

\section{Consent}

Informed consent was waived due to the study's retrospective design.

\section{Disclosure}

The authors had full access to the database and take full responsibility for its integrity.

\section{Conflicts of Interest}

The authors declare that they have no conflicts of interest.

\section{Authors' Contributions}

All authors have read and agreed to the manuscript as written.

\section{Acknowledgments}

This research was supported by the Bio \& Medical Technology Development Program of the NRF funded by the Korean government, MSIP (2015M3A9B6029139).

\section{References}

[1] K. Chigurupati, L. Reshmi, S. Gadhinglajkar, S. Venkateshwaran, and R. Sreedhar, "Pulmonary edema following transcatheter closure of atrial septal defect," Annals of Cardiac Anaesthesia, vol. 18, no. 3, pp. 441-444, 2015.

[2] P. Wagdi, "Incidence and predictors of atrial fibrillation following transcatheter closure of interatrial septal communications using contemporary devices," Clinical Research in Cardiology, vol. 99, no. 8, pp. 507-510, 2010.

[3] Y. Takaya, T. Akagi, Y. Kijima, K. Nakagawa, S. Sano, and H. Ito, "Long-term outcome after transcatheter closure of atrial septal defect in older patients," JACC: Cardiovascular Interventions, vol. 8, no. 4, pp. 600-606, 2015.

[4] A. S. Levey, L. A. Stevens, C. H. Schmid et al., "A new equation to estimate glomerular filtration rate," Annals of Internal Medicine, vol. 150, no. 9, pp. 604-612, 2009.

[5] R. M. Lang, M. Bierig, R. B. Devereux et al., "Recommendations for chamber quantification: a report from the American society of echocardiography's guidelines and standards committee and the chamber quantification writing group, developed in conjunction with the European association of echocardiography, a branch of the European society of cardiology," Journal of the American Society of Echocardiography, vol. 18, no. 12, pp. 1440-1463, 2005.

[6] J. M. Brennan, J. E. Blair, S. Goonewardena et al., "Reappraisal of the use of inferior vena cava for estimating right atrial pressure," Journal of the American Society of Echocardiography, vol. 20, no. 7, pp. 857-861, 2007.

[7] A. Y. Her, J.-Y. Kim, E.-Y. Choi et al., "Value of ventricular stiffness index and ventriculoarterial interaction in patients with nonischemic dilated cardiomyopathy," Circulation Journal, vol. 73, no. 9, pp. 1683-1690, 2009.

[8] Z. Sasson, P. G. Yock, L. K. Hatle, E. L. Alderman, and R. L. Popp, "Doppler echocardiographic determination of the pressure gradient in hypertrophic cardiomyopathy," Journal of the American College of Cardiology, vol. 11, no. 4, pp. 752-756, 1988.

[9] A. C. Boyd, M. Cooper, and L. Thomas, "Segmental atrial function following percutaneous closure of atrial septum using occluder device," Journal of the American Society of Echocardiography, vol. 22, no. 5, pp. 508-516, 2009.

[10] Y. H. Kong, J. Song, K. H. Kim, J. Huh, and I. S. Kang, "Acute changes in left ventricular end diastolic pressure following the transcatheter closure of an atrial septal defect in adults," The Heart Surgery Forum, vol. 19, no. 3, pp. E145-E149, 2016.

[11] M. Hanninen, A. Kmet, D. A. Taylor, D. B. Ross, I. Rebeyka, and I. F. V. Muhll, "Atrial septal defect closure in the elderly is associated with excellent quality of life, functional improvement, and ventricular remodelling," Canadian Journal of Cardiology, vol. 27, no. 6, pp. 698-704, 2011. 